\title{
Practice-based research network solutions to methodological challenges encountered in a national, prospective cohort study of mothers and newborns
}

\author{
Stacia A. Finch ${ }^{a}$, Christina Lalama ${ }^{b}$, Cathie Spino ${ }^{d}$, Heidi C. Schwartz ${ }^{a}$, Richard C. Wasserman ${ }^{a, e}$, Marie C. McCormick $^{c}$ and \\ Henry H. Bernstein ${ }^{f}$ \\ aPediatric Research in Office Settings (PROS), Department of Research, American Academy of Pediatrics, Elk Grove Village, IL, Departments of \\ ${ }^{b}$ Biostatistics and ${ }^{c}$ Maternal and Child Health, Harvard School of Public Health, Boston, MA, ${ }^{d}$ Department of Biostatistics, University of \\ Michigan, Ann Arbor, MI, ${ }^{e}$ Department of Pediatrics, University of Vermont, Burlington, VT, and ${ }^{f}$ General Pediatrics, Dartmouth Medical \\ School, Children's Hospital at Dartmouth, Lebanon, NH, USA
}

Correspondence:

Henry H. Bernstein, D.O.,

Chief, General Academic

Pediatrics, Dartmouth

Hitchcock Medical Center,

One Medical Center Drive,

Lebanon, NH 03756, USA.

E-mail:

henry.bernstein@hitchcock.org

\section{Summary}

Finch SA, Lalama C, Spino C, Schwartz HC, Wasserman RC, McCormick MC, Bernstein $\mathrm{HH}$. Practice-based research network solutions to methodological challenges encountered in a national, prospective cohort study of mothers and newborns. Paediatric and Perinatal Epidemiology 2008; 22: 87-98.

The emergence of practice-based research networks (PBRN) has facilitated the execution of multifaceted community-based studies. As study complexity increases, so does the number of methodological barriers encountered. This paper's goal was to delineate methodological barriers and to evaluate the effectiveness of selected strategies and approaches developed and implemented in allowing a prospective, national PBRN cohort study to succeed in enrolling geographically dispersed mother/healthy term infant dyads $(n=4300)$ on the day of post-partum discharge. Specific methodological barriers included: (1) obtaining multiple Institutional Review Board (IRB) approvals; (2) gathering longitudinal data from multiple individuals; (3) soliciting multiple perspectives on discharge decision making; and (4) bolstering minority enrolment. The most effective strategies and approaches we employed to address these methodological challenges were: (1) preparing and distributing the 'IRB Packet'; (2) recruiting multiple practices covered by the same IRB; and (3) obtaining supplemental funding for increasing minority enrolment. We expect that other PBRN investigators can benefit from our experience and solutions in the successful conduct of this multifaceted community-based study.

Keywords: enrolment strategies, community studies, ethnic minority, study methodology.

\section{Introduction}

Conducting complex national studies in geographically dispersed settings presents many challenges. The emergence of practice-based research networks (PBRN) - 'groups of ambulatory practices devoted principally to the primary care of patients, affiliated with each other in order to investigate questions related to community-based practice ${ }^{11}$ - has enabled researchers to more easily access samples from communities across a single country. In 2000, 2001, and 2002 the Agency for Healthcare Research and Quality awarded infrastructure grants to several PBRNs in maternal and child health. In addition, the growing number of publications by various $\mathrm{PBRNs}^{2-6}$ illustrate their rising importance.

Pediatric Research in Office Settings (PROS) is a national paediatric PBRN that was established by the American Academy of Pediatrics (AAP) in $1986^{7}$ and sustained, in part, by core funding from the Maternal and Child Health Bureau/Health Resources and Services Administration. PROS strives to accurately represent the AAP's membership., ${ }^{2,8}$ PROS members and researchers work closely together to generate research questions, design study materials and 
protocols, obtain funding, collect and analyse study data, and publish results. PROS has studied diverse child health topics, generating new knowledge that has led to changes in the clinical guidelines of the AAP and other organizations. ${ }^{2,9-11}$ PROS developed the Life Around Newborn Discharge (LAND) Study to address gaps in knowledge on factors related to post-partum discharge timing and to provide information 'needed to inform policy on reimbursement and clinical care for the general population. ${ }^{12-19}$ Based on input from PROS practitioners, the LAND Study investigators sought to go beyond prior research restricted to sentinel events (e.g. hospitalisations related to length of stay $)^{20}$ to examine the determinants and impact of joint decision making about post-partum discharge, as emphasised in the Newborns' and Mothers' Health Protection Act of 1996. ${ }^{21}$ This report describes methodological barriers (and solutions) in conducting complex national studies in geographically dispersed settings, using the LAND Study as a case example.

LAND's specific study aims were to: (1) describe readiness of mother and newborn for post-partum hospital discharge based upon maternal, paediatric, and obstetric perceptions and clinical judgements; (2) explore determinants of readiness; and (3) examine the relationship between maternal, paediatric, and obstetric perceptions and clinical judgements concerning mother and infant readiness for post-partum hospital discharge and subsequent health care utilisation, health-related behaviours, and infant and maternal health status during the first 4 weeks after discharge. Factors in decision making for post-partum discharge were critical to LAND's aims because discharge when a family is neither medically nor psychologically prepared places the new family at greater risk of poor maternal or infant health outcomes, increased use of health services, or adoption of behaviours disadvantageous to maternal and infant health during the immediate post-partum period. ${ }^{3}$ In the course of this research, we encountered several obstacles that could have resulted in the inability to: (1) reach enrolment goals for numbers of practices and/or mother/infant dyads; (2) assess readiness for discharge perceptions directly from the three parties; and (3) examine vulnerable subgroups because of the modest enrolment of a diverse national sample in terms of racial, ethnic, and socio-economic backgrounds.

This paper's goal was to delineate methodological barriers and to evaluate the effectiveness of selected strategies and approaches developed and implemented in allowing a prospective, national PBRN cohort study to succeed. We expect that other PBRN investigators can benefit from our experience and solutions.

\section{Methodological challenges}

\section{Multiple Institutional Review Board (IRB) approvals}

Subject enrolment and data collection by multiple practitioners in geographically dispersed hospitals presented challenging IRB logistics. In previous outpatient-only PROS studies, independent practices not owned by institutions had no local IRBs from which to obtain approval, allowing the AAP's IRB alone to serve this purpose. However, as LAND subjects were recruited in hospitals, all practices required local IRB approval to conduct data collection at these sites. Researchers have found that local IRBs can vary considerably on important issues, even when assessing identical clinical trial protocols. ${ }^{22}$ Few IRBs present a clear instruction for investigators who seek to design ethically appropriate studies. ${ }^{23}$ IRB review processes at different sites may be divergent enough to prevent national clinical research projects from obtaining approval in a timely and efficient manner, ${ }^{24}$ which could pose a threat to the study's sample size in terms of participating practices and patients.

\section{Complex longitudinal data collection}

The LAND Study was a prospective observational cohort design with a longitudinal follow-up period of 4 weeks. It was the first inpatient study, as well as the most difficult, conducted by PROS. Collecting data on nine self-administered questionnaires from multiple individuals (mother, paediatrician, obstetrician) in multiple settings (hospital, paediatric office, home) at several points in time (day of post-partum discharge, at each ambulatory office encounter of the infant with the paediatric clinician, first 14 days after post-partum discharge by mother, and 4 weeks after post-partum discharge) was challenging but necessary to achieve the study's goals. Securing a clear logistical connection between each hospital enrolment site and the subsequent ambulatory clinical care site was vital in facilitating communication between clinical settings and improving long-term monitoring and tracking of patients. 
In order to describe readiness of mother and newborn for post-partum hospital discharge based on perceptions of all individuals, each party completed the surveys on the day of post-partum discharge. Obtaining information from mothers post-discharge was necessary and required the tracking of mailed-in surveys completed over 4 weeks. Collecting data longitudinally allowed the team to assess changes in discharge decision making as well as health care utilisation from the mother and paediatrician. Because minimising the impact of recall bias on short-term maternal and infant morbidity constituted a priority, mothers received a daily diary to complete for the first 14 days after post-partum discharge. Diaries represent an effective means of obtaining detailed and accurate personal data from respondents, ${ }^{25-28}$ and the daily, close-ended home recording tool has yielded important clinical data in studies of asthma, pharyngitis, mental health, pain, crying patterns, and quality of life..$^{29-32}$ A 2-week interval offers advantages over longer periods. ${ }^{33}$ When this system of data collection, accompanied by clear instructions and linked with reminders such as telephone prompts, is used for relatively short periods, it produces higher response rates than those documented with either mail or telephone surveys. ${ }^{26}$ Compared with an exit survey, diaries may provide a more complete and accurate recall of relatively minor concerns. ${ }^{34}$ Multiple participants from several settings collaborated to ensure that information was obtained at the time of discharge and to prevent loss to follow-up at the 4-week interview.

\section{Multiple perspectives on discharge decision making}

Ideally, readiness for discharge should be determined by consensus among the mother, her partner, the obstetrician, and the paediatrician, as highlighted by federal legislation. ${ }^{35}$ Previously, PROS had never attempted to collect data from obstetricians and non-PROS members, but felt it was necessary to include all practitioners. This decision required methodological innovation. The involvement of non-PROS practitioners was essential because not all PROS members are responsible for the care of newborns in the hospital nursery, which precluded them from enrolling dyads at the hospital. Hospital-based paediatricians possessed the ability to obtain the consent of and enrol the mothers as well as complete the initial questionnaire. The goal was to collect data on perceptions of readiness for mother/ infant discharge from the mother and from hospital- based paediatricians and obstetricians. Because these practitioners are not PROS members, a concerted effort was necessary to ensure their data were obtained.

\section{Minority enrolment}

Recruitment of minorities poses a recognised challenge in clinical research ${ }^{36,37}$ and, especially, in PROS studies (i.e. study minority patient samples range from $13 \%^{8}$ to $26 \%{ }^{4}$ ). Lack of a diverse study sample threatens the ability to generalise the results and to examine vulnerable subgroups.

\section{Methods}

\section{Sample}

PROS afforded the LAND Study access to a national sample of US paediatric practices and families, enhancing generalisability while accounting for state-to-state variability in terms of discharging practices. Prior to data collection, participating practices reviewed a detailed instruction manual that described the study protocol at each site and the timeline for survey completion. Participating practitioners identified eligible mother/infant dyads by reviewing their daily hospital-generated list of newborn babies in the nursery. They approached the mothers regarding participation on the day of hospital discharge. If the mother agreed, written informed consent was obtained and study materials (written at a 4th-6th grade reading level) were distributed. The study protocol required each practice to enrol all eligible newborns seen consecutively in the hospital nursery during a period of at least 8 weeks, including weekends and holidays, or until they enrolled 120 pairs ( $2 \%$ of targeted sample size). Practices were also asked to remind mothers to complete the longitudinal surveys by: (1) a phone call 1-2 days following hospital discharge to encourage completion and return of the diary; and (2) a mailed postcard 3 weeks after discharge as a reminder to complete the follow-up questionnaire.

Access to hundreds of practices allowed for a large sample size and afforded the possibility of studying important subgroups (e.g. minorities and younger mothers) for whom differences in discharge decision making and outcomes might exist. More than 750 PROS practices serve approximately three million of the nation's children ${ }^{38}$ and currently include over 2000 practitioners from 49 states, District of Columbia, 

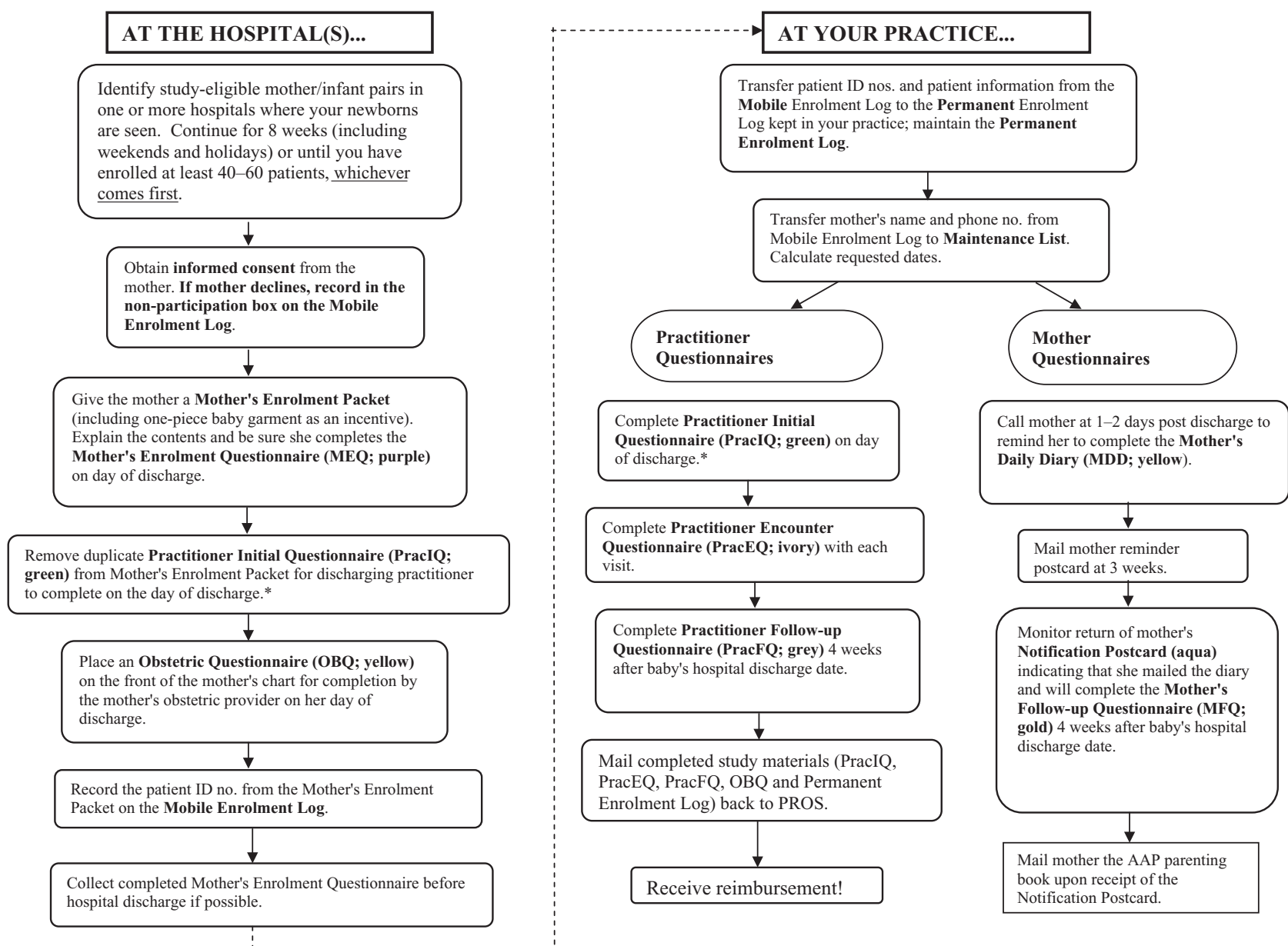

Figure 1. Life Around Newborn Discharge patient enrolment overview. *PracIQ can be completed at the hospital or at your practice on the day of discharge. AAP, American Academy of Pediatrics.

Canada and the Commonwealth of Puerto Rico. PROS members include paediatricians, family practitioners, nurse practitioners, and physician assistants. However, previous PROS experience suggested that relying solely on the usual recruitment strategies (e.g. inviting existing PROS practices to participate) might not provide sufficient power to examine the experience of minority women because PROS has a limited number of practices that serve a large number of minority patients. This required PROS to develop additional strategies to enhance the sample with practices serving large minority populations.

\section{Design}

Methods used in gathering and analysing information presented in this paper are documented in an extensive database, including PROS staff tracking practice recruitment data, IRB specifics, problems encountered, strategies suggested and implemented, and results. The distribution of monthly progress reports that detailed practice and dyad recruitment, including minority enrolment and IRB approval status, allowed each study team member to be updated concerning the overall status. Monthly study team conference calls allowed discussion of various strategies for improving specific areas to reach goals and to make decisions regarding implementation. The database contains records of all communications with practices that were invited to participate. Most frequently reported challenges were selected for inclusion in this paper. Strategies were considered successful if they reduced or eliminated threats to the study and/or increased practice and dyad enrolment rates. 


\section{Strategies and experience}

\section{Multiple IRB approvals}

Many PROS members were unfamiliar with the IRB approval process, so the LAND study team had to determine strategies for facilitating this process for each individual practice.

LAND recruitment was conducted by practice rather than by individual practitioner. A practice was eligible to participate if it could: (1) obtain local IRB approval; (2) conduct enrolment through PROS practitioners or non-PROS newborn nursery colleagues who were responsible for the care of newborns in the hospital nurseries, enrolling mother/infant dyads who would be seen for follow-up visits in a PROS practice; and (3) identify an office research coordinator to obtain written consent from mothers at the hospital, and maintain and complete the paperwork involved in outpatient (ambulatory) follow-up data collection.

The local IRB approval processes were facilitated by the development of a comprehensive 'IRB Packet.' It contained all necessary information on a computer disk that could easily be copied onto the local application form. If practitioners were still unable to complete the local IRB process, the study team completed the entire application for the practice and communicated directly with the IRB administrator until final approval was secured at each local site. To minimise the number of IRBs involved, we recruited multiple eligible practices (both PROS and non-PROS) covered by the same local IRB.

\section{Complex longitudinal data collection}

Data collection required new tactics to improve the interface between the hospital and office visit site, and to track data from multiple individuals across time. Figure 1 depicts a summary of the enrolment strategy.

Enrolling practitioners completed a 'Mobile Enrolment $\log ^{\prime}$ that tracked eligible dyads in the nursery and indicated whether or not they were enrolled in the hospital. This log was forwarded to the practitioner's practice, where information was transferred to the 'Permanent Enrolment Log' for the follow-up portion of the study in the ambulatory setting.

Each practice ensured complete and accurate information transfer by designating an office research coordinator. Participating paediatricians served in this role in some practices, while other practices paid a specific office staff member with an incentive of $\$ 30$ per enrolled dyad.

As a participation incentive, mothers received a onepiece baby garment with a printed LAND logo at the time of enrolment (hospital discharge) and the AAP's book, Caring for Your Baby and Young Child: Birth to Age 5 , after completing and returning the Mother's Daily Diary.

\section{Multiple perspectives on discharge decision making}

\section{Obstetric informants}

To enhance the participation of obstetricians in the study, we consulted with obstetric consultants and included the American College of Obstetricians and Gynecologists' (ACOG) endorsement of the study on the back of the Obstetric Questionnaire. The ACOG Collaborative Ambulatory Research Network ${ }^{39}$ confines itself to survey studies and does not collect data directly from patients; therefore, LAND investigators did not have access to a clinically experienced obstetric PBRN. A number of participating practices implemented various strategies (e.g. PROS practitioners attended obstetric/gynaecology staff meetings, distributed candy/coffee vouchers), which were also suggested to other participants when they indicated a low obstetrician response.

\section{Non-PBRN practitioner participation}

Participating PROS practitioners who were not responsible for the care of newborns in the nurseries were instructed to approach the hospital-based staff (e.g. covering paediatricians, house staff) regarding collaborating on mother/infant enrolment in the hospital.

\section{Minority enrolment}

In order to ensure a diverse national sample in terms of racial, ethnic, and socio-economic backgrounds and allow for analysis of vulnerable subgroups, the study team's goal was to enrol $25 \%$ minority and disadvantaged children into the study. In addition, all maternal questionnaires were made culturally sensitive for African American and Latino populations and translated into universal-dialect Spanish (with backtranslation for quality control). The literature suggests that the use of multiple strategies and financial compensation significantly improve the attainment of 
minority recruitment goals. ${ }^{36,37,40-42}$ The aim was to recruit practices serving a high percentage (defined as $\geq 40 \%$ ) of minority patients (defined as either nonwhite and/or Hispanic). Because PROS has a limited number of practices that serve a large percentage of minority patients (only 20\% of those recruited) and because of the emergence of a differential refusal rate (61\% of practices serving a high percentage of minority patients vs. $39 \%$ of other practices), the study team sought to identify new additional, previously nonPROS practices to help meet the study's minority enrolment goal.

A multi-pronged approach was implemented to recruit additional practices serving minority populations. This included: (1) partnering with a non-profit organisation comprised of practices serving high percentages of African American and Hispanic populations; (2) challenging PROS leadership to recruit at least one non-PROS practice serving minority populations in their state; (3) distributing study information at various $\mathrm{AAP}$ meetings attended by members and the leadership; (4) publishing an AAP News article specifically calling for paediatricians who served inner-city populations to participate; and (5) acquiring a supplemental grant (\$105 per enrolled dyad) to focus practice efforts on developing effective local strategies for the recruitment of minority dyads and for coordinating data collection.

\section{Results}

By addressing the methodological challenges with these specific strategies, we were able to enrol a large sample of geographically diverse healthy mother and infant dyads at the time of post-partum discharge.

\section{Multiple IRB approvals}

\section{Recruitment of practices/sample}

Of the 620 PROS practices invited to participate in LAND, 132 (21\%) agreed to participate while 302 practices $(49 \%)$ declined for several reasons (e.g. absence of a practitioner responsible for the care of newborns in the nursery, the time-consuming nature of the IRB process, inability to identify an office research coordinator). This participation rate was lower than that of other completed PROS studies of similar size (range $30-38 \%$ ). Of the 132 practices agreeing to participate, 20 $(15 \%)$ discontinued participation without enrolling any dyads. Reasons for dropout without collecting data included the inability of obtaining local IRB approval $(5 \%)$ and deciding that the study would be too difficult to complete in their practice setting (95\%). The remaining 112 practices with 451 practitioners in 35 states contributed data for eligible dyads between September 1999 and August 2002. (See Acknowledgements section for a complete list of participating practices by AAP chapter.)

The number of participating practitioners per practice ranged from 1 to 18 . Practices were diverse in organisation and setting; participating PROS practices did not differ significantly from non-participating ones in terms of type (e.g. one-third of each group consisted of $\geq 3$ paediatric providers) or location (e.g. $40 \%$ of each group practiced in an urban area). Comparison of par-

Table 1. Maternal/infant dyad characteristics

\begin{tabular}{|c|c|}
\hline & Summary ${ }^{a}$ \\
\hline \multicolumn{2}{|l|}{ Maternal characteristics } \\
\hline First baby & $41 \%(1437)$ \\
\hline Vaginal delivery & $80 \%(1948)$ \\
\hline Maternal education $<$ high school & $18 \%(621)$ \\
\hline Maternal age $<18$ years & $3 \%(90)$ \\
\hline Mean maternal age, years & $27.5 \pm 6.0^{\mathrm{b}}$ \\
\hline Marital status (parents living together) & $87 \%(3055)$ \\
\hline \multicolumn{2}{|l|}{ Income } \\
\hline$<\$ 40 \mathrm{~K}$ & $50 \%(1539)$ \\
\hline$\$ 40 \mathrm{~K}$ to 59999 & $18 \%(550)$ \\
\hline$\$ 60 \mathrm{~K}+$ & $32 \%(984)$ \\
\hline \multicolumn{2}{|l|}{ Insurance source } \\
\hline Private & $61 \%(1249)$ \\
\hline Public & $36 \%(720)$ \\
\hline Uninsured & $3 \%(65)$ \\
\hline \multicolumn{2}{|l|}{ Prenatal care } \\
\hline Received prenatal care during pregnancy & $94 \%(3262)$ \\
\hline Attended any prenatal class & $36 \%(1253)$ \\
\hline Prenatal problems & $18 \%(688)$ \\
\hline $\begin{array}{l}\text { Health problems requiring extra visits/tests } \\
\text { during pregnancy }\end{array}$ & $38 \%(1288)$ \\
\hline Met paediatrician while pregnant & $21 \%(748)$ \\
\hline \multicolumn{2}{|l|}{ Plan to feed baby at home by ... } \\
\hline Bottle & $25 \%(897)$ \\
\hline Breast & $63 \%(2217)$ \\
\hline Both & $12 \%(419)$ \\
\hline \multicolumn{2}{|l|}{ Infant race/ethnicity } \\
\hline White, non-Hispanic & $62 \%(2408)$ \\
\hline Black, non-Hispanic & $12 \%(447)$ \\
\hline Hispanic & $21 \%(812)$ \\
\hline Other, non-Hispanic & $5 \%(194)$ \\
\hline
\end{tabular}

aProportion (no.) with the characteristic.

${ }^{\mathrm{b}}$ Mean \pm Standard Deviation. 
ticipating PROS practitioners with a subset of AAP members indicated similarity of age and gender but difference by practice area, with more PROS practitioners tending to practice in rural ( 25 vs. $14 \%$ ) than in suburban areas ( 38 vs. $54 \%$ ). ${ }^{43}$ This PROS group also differed from the AAP subset in practice arrangement by practising less in paediatric groups (36 vs. $45 \%$ ).

Table 1 summarises the characteristics of the enrolled maternal/infant dyads. In order to increase the study's sample size, 20 practices $(18 \%)$ completed a second enrolment period of at least 8 weeks, with overall total enrolment per practice limited to no more than 120 dyads. The number of dyads enrolled by participating practices ranged from 1 to 120 , with most $(60 \%)$ enrolling between 6 and 40 .

\section{Creation of 'IRB Packet' and completion of IRB process by study team}

Local IRB approval was obtained for the 112 participating practices at 78 different hospitals, of which 12 deemed the AAP's central IRB approval as sufficient. The local IRB approval process took an average of 96 days to complete from the time a practice received the comprehensive 'IRB Packet' from PROS staff to receiving final approval. Local IRBs required minimal modifications (e.g. additions to contact names/phone numbers, approval stamps); none required modifications that substantively changed the study protocol. Most practices (78\%) found the 'IRB Packet' quite useful; however, 23 practices felt that the IRB process remained too time-consuming and chose not to participate. PROS staff facilitated the process for five practices.

\section{Recruitment of multiple practices covered by the same IRB}

Approaching multiple practices covered by the same local IRB was an effective strategy in at least four states, proving easier and less time-consuming than obtaining approval at what otherwise might have been 27 separate hospitals rather than four.

\section{Complex longitudinal data collection}

\section{Creation of 'Mobile Enrolment Log'}

The 'Mobile Enrolment Log' proved to be an accurate tracking device for eligible dyads. Analysis indicated that 4300 of 4974 eligible mother/infant dyads (86\%) enrolled into the study. The reasons reported for maternal non-participation included maternal refusal $(n=333)$ and the provider's inability to approach the mother $(n=126)$. A miscellaneous/other category was noted for 215 others, but details were not collected. Of the 112 participating practices, $47(42 \%)$ reported that all eligible dyads approached enrolled into the study; the remaining practices reported from 1 to 75 maternal non-participants per site.

Due to complex longitudinal data collection, differences were found in the completion of the four-week follow up questionnaire. Mothers who did not complete the follow-up questionnaire were younger, less

\begin{tabular}{lcc}
\hline LAND questionnaire & $\begin{array}{c}\text { Number of } \\
\text { questionnaires } \\
\text { (eligible dyads only) }\end{array}$ & $\begin{array}{c}\text { Percentage of } \\
\text { overall enrolled } \\
(n=4300)\end{array}$ \\
\hline Mother's enrolment questionnaire & 3551 & $83 \%$ \\
Mother's daily diary & 2893 & $67 \%$ \\
Mother's follow-up questionnaire & 2554 & $59 \%$ \\
Obstetric questionnaire & 2778 & $65 \%$ \\
Practitioner initial questionnaire & 4078 & $95 \%$ \\
Practitioner encounter questionnaire: & 3782 & $88 \%$ \\
$\quad$ number that had at least one encounter & (total completed: 6831) & $90 \%$ \\
reported & 3890 & $26 \%$ \\
Practitioner follow-up questionnaire & 1113 & $50 \%$ \\
Number of dyads with all questionnaires & 2141 & \\
Number of dyads with all questionnaires & & \\
$\quad$ except obstetric questionnaire & & \\
\hline
\end{tabular}

Table 2. Questionnaire response rates for the full study cohort

LAND, Life Around Newborn Discharge. 
educated, and seen by younger paediatricians than those mothers who completed the questionnaire.

\section{Designate office research coordinator}

Designation of the practice incentive for the sole purpose of 'hiring' a specific office staff member to support the interface between the office and the hospital proved a useful strategy in eight Vermont and six Illinois practices; the nurses enrolled 255 and 209 dyads, respectively. Other sites engaged volunteer medical students to serve in this position; one California private practice enrolled 104 dyads by this method.

\section{Multiple perspectives on discharge decision making}

\section{Obstetric participation}

Table 2 summarises response rates for all questionnaires. Unfortunately, endorsement of the project by ACOG did not promote completion of study materials by the obstetricians as anticipated. Only $65 \%$ of obstetricians completed their survey at the time of discharge, while $85 \%$ of mothers and $95 \%$ of paediatric practitioners did so. Furthermore, participating PROS practitioners attending obstetric/gynaecology staff meetings and distributing candy/coffee vouchers resulted in only modest participation in a few practices.

To compensate for obstetric non-response, primary analyses of the study are being conducted by using multiple imputation techniques. ${ }^{44}$ Multiple imputation inserts plausible values (that are consistent with the rest of the data as determined by an imputation model) for missing data to create a number of complete data sets. Then, application of standard statistical analyses on each complete data set produces estimates that are combined to yield one set of estimates and standard errors that appropriately preserve the uncertainty associated with 'filling in' missing data.

\section{Non-PBRN practitioner participation}

The inclusion of non-PROS hospital-based staff, such as house staff and covering paediatricians, to participate in the enrolment phase was deemed successful based on the number of PROS practices that otherwise would not have been able to participate as a result of not being responsible for the care of newborns in the nursery. Of the 4078 practitioner questionnaires completed at the time of enrolment, non-PROS practitio- ners completed $867(21 \%)$ of them, indicating that those dyads were enrolled by hospital-based practitioners covering at the hospital for PROS colleagues.

\section{Minority enrolment}

\section{Funding for practices serving minority patients}

The supplemental grant strategy achieved the greatest success in increasing minority enrolment and assisted in contributing $33 \%$ of the total study sample with minority patients (e.g. $12 \%$ black and non-Hispanic, $21 \%$ Hispanic). These additional funds helped to defray at least some of the costs (e.g. staff time) incurred by participating practices. Although these additional funds did not completely reimburse practices for their work, the financial support was instrumental for some in hiring a person who could serve as an 'office research coordinator' or in identifying a practitioner at the hospital who was responsible for the care of newborns in the nursery. The ten practices (36\% of participating practices serving a high percentage of minority patients) identified by PROS to receive additional funds for participation enrolled 409 mother/infant dyads ( $10 \%$ of the total study sample). Of these, 354 dyads $(87 \%)$ were minorities, which represented almost one-quarter $(24 \%)$ of the total minority sample.

\section{Remaining strategies of multi-pronged approach}

The other strategies implemented to increase the number of enrolled practices serving minorities (e.g. distributing information at AAP meetings, publishing an $A A P$ News article) were not as effective in increasing sample size but still provided an additional 151 practices that could be recruited. Twenty-eight $(19 \%)$ of these joined PROS, with eight of them completing the LAND study. Five of these practices had been identified by the PROS leadership as part of the challenge strategy to recruit a non-PROS practice in each state. Unfortunately, the strategy of partnering with a nonprofit organisation comprised of practices serving high percentages of African American and Hispanic populations was unsuccessful, as no practices recommended by this organisation participated in data collection. 


\section{Making maternal questionnaires culturally sensitive}

In addition to providing patients with incentives, PROS collaborated with a national non-profit organisation, whose mission is minority health improvement, to create culturally sensitive questionnaires. Team members and consultants reviewed each questionnaire for content and consistency within and between instruments, as well as for cultural sensitivity. Spanish-speaking PROS practitioners and mothers from multiple Latino cultures then reviewed the Spanish materials at a variety of reading levels to ensure that the instruments were understandable across cultural perspectives. Utilising Spanish questionnaires was considered successful as 44 practices received Spanish materials and 26 of them enrolled 317 dyads. A projected number of completed Spanish questionnaires was not established; however, the overall goal was to increase the minority dyad enrolment by allowing as many mothers as possible to participate.

\section{Discussion}

This prospective, national cohort study conducted in a PBRN enrolled the largest sample to date of geographically dispersed mother/healthy term infant dyads $(n=4300)$ on the day of post-partum discharge. The PROS network provided a unique laboratory through which to study post-partum newborn discharge, with the idea originating from a practicing paediatrician and its research questions, study design, and methods being developed with the input of at least 100 paediatricians in active clinical practice.

The LAND Study's goal was to describe readiness of mother and newborn for discharge based on perceptions of mother, paediatrician, and obstetrician, and to determine the effect of this decision-making pattern on a broad array of potential outcomes. Many challenges were identified (e.g. multiple IRB approvals, complex longitudinal data collection, multiple perspectives on discharge decision making, minority enrolment) that threatened to undermine the study's success by limiting practice and dyad sample sizes, ability to collect critical data, and generalisability. Although multiple strategies were implemented in an attempt to overcome the challenges of this study, some ultimately proved more effective than others. The most successful strategies (defined as reducing or eliminating study threats and/or increasing practice and dyad enrolment rates) were: (1) preparing and distributing the 'IRB Packet'; (2) recruiting multiple practices covered by the same IRB; and (3) obtaining supplemental funding for increasing minority enrolment.

Other strategies were considered less successful in that they did not increase sample size and response rate, resulting in the study team's need to use more complex statistical techniques to address missing data. Additional strategies might have been implemented to improve obstetrician participation, such as distribution of specific incentives to the obstetricians or hospital department or by obtaining study endorsement through their obstetric directors. The mother's follow-up questionnaire response rate was also less than optimal, but would have required additional work by practices (e.g. completing phone interviews as evidenced by Escobar et al. ${ }^{45}$ achieving an $80 \%$ response rate) or more incentives. Lastly, the non-profit organisation's efforts were largely ineffectual because of the lack of clinical research experience in their minority-serving practices. The study team addressed the lack of success with these strategies by implementing alternative strategies.

Applying lessons learned, PROS has adopted some of the LAND Study strategies in subsequent national studies, especially: (1) recruiting multiple practices covered by the same IRB; (2) allowing non-research network practitioners to participate; (3) adding consultants from other disciplines to study teams; and (4) finding additional funds to support practices serving a high percentage of minority patients at enhanced rates. The utilisation of these specific strategies in large, methodologically complex studies should be beneficial in eliminating similar major challenges to network research.

Advancing technology may also provide new solutions to the challenges encountered in this study. For example, one possible way to improve both IRB efficiency in reviewing clinical research projects and hospital and office interface is a web-based query and notification system (QNS). ${ }^{46}$ The QNS creates an interactive flow of reliable information among site coordinators, protocol teams, and regulatory groups, thus ensuring a more timely way to communicate accurate responses to queries and reports. Additionally, a QNS can be created at minimal cost with already existent web-based devices, making it a feasible possibility in future national clinical research projects. 


\section{Study limitations}

How generalisable are results from studies carried out in PBRNs? It is important to note that practitioners in PROS are volunteers. When paediatricians participate in research, time spent on study-specific tasks is time spent away from patient care. In addition, PROS experience suggests that not all paediatricians are willing to serve as an office research coordinator or to allow personnel to do so for minimal reimbursement that does not cover time and effort. It is unclear, however, whether the volunteer status of study practitioners would make the mothers and newborns in their practices less representative of mothers and newborns in general, or whether it would lead practitioners to overestimate or underestimate unreadiness for postpartum discharge.

In addition, restricted access to care prevented certain patient populations from participating in this study. Our results may thus not be generalisable to these subpopulations excluded from the study, but the inclusion of all underserved populations in such a complex study would have been logistically and financially impractical. Finally, we had limitations in reporting the specific breakdown of a number of practices for each refusal reason for participation as not all practices communicated this information to PROS. Certain practices specifically provided to PROS the examples cited in the text.

\section{Conclusion}

Using a national PBRN, the LAND Study enrolled the largest sample ever of geographically dispersed mother/healthy term infant dyads on the day of postpartum discharge. The strategies employed to overcome methodological challenges in the successful conduct of this large, national, observational cohort study should provide valuable guidance to future clinical investigators.

\section{Acknowledgements}

This study was supported by grants from the Health Resources and Services Administration Maternal and Child Health Bureau (\#5 R40 MC00117 and 2R60 MC 00107-09) with additional support from the AAP's Friends of Children and Research in Pediatric Practice Fund. The superb administrative assistance of Holly Desrosiers and Grace Yang in preparing this manuscript for submission is also much appreciated.
We especially appreciate the efforts of the PROS practices and practitioners. The paediatric practices or individual practitioners who enrolled mother/infant dyads in this study are listed here by AAP Chapter: Alabama: University of South Alabama (Mobile); Alaska: Anchorage Pediatric Group (Anchorage); Arizona: Orange Grove Pediatrics (Tucson); California-1: Castro Valley Pediatrics (Castro Valley), Razia Sheikh, MD (Fresno), Drs Cantor, Giedt, Kamachi \& Brennan (Salinas), UCSF - Laurel Heights (San Francisco); California-2: UCLA West Los Angeles Office (Los Angeles); California-3: Pediatric Medical Associate (Vista); Colorado: Rocky Mountain Youth Medical Providers, PC (Thornton); Connecticut: Healthwide Medical Associates (Vernon Rockville); East Military: National Naval Medical Center (Bethesda); Florida: Family Health Center East \& Women and Children's Health Center (Orlando), ORH Pediatric Outpatient Clinic (Orlando), Sacred Heart Pediatric Care Center (Pensacola), Kenneth S Cohen, MD (Pembroke Pines); Georgia: Decatur Pediatric Group (Clarkston), Snapfinger Woods Pediatric Associates, PC (Decatur), The Pediatric Center (Stone Mountain), Darshan S Shah, MD (Swainsboro); Hawaii: Island Youth Heart and Health Center (Hilo), University of Hawaii (Honolulu); Illinois: Sinai Health System Hawthorne Works (Cicero), Kidz Health (Chicago), Kedzie Plaza Sinai Medical Group (Chicago), Sinai Health System - Centro Medico San Rafael (Chicago), Sinai Health System - Centro Medico SMG (Chicago), Sinai Health System - Kling Pediatrics (Chicago), Sinai Health System - Plaza Medical Center (Chicago); Indiana: Southern Indiana Pediatrics (Bedford), Southern Indiana Pediatrics, LLC (Bloomington), IU Medical Group (Indianapolis), Saint Vincent Pediatric Clinic (Indianapolis), Marshall County Pediatrics (Plymouth); Iowa: River Valley Healthcare Pediatrics (Bettendorf), University of Iowa (Iowa City); Kansas: Ashley Clinic (Chanute), Bethel Pediatrics (Newton); Maine: Kennebec Pediatrics (Augusta); Maryland: Shore Pediatrics (Easton), Main Street Pediatrics, LLC (Towson), Shady Side Medical Associates (Shady Side); Massachusetts: Alewife Brook Community Pediatrics (Arlington), Burlington Pediatrics (Burlington), Mystic Valley Pediatrics (Medford), Mary Lane Pediatric Associates (Ware), University of Massachusetts Memorial Pediatric Primary Care (Worcester); Michigan: Pediatric \& Adolescent Medicine (Bay City); Minnesota: Kundel \& Streitz, MD, PA (Duluth); Missouri: MMPG Physicians Group (Kansas City), Northland Pediatric Associates (North Kansas City), Timothy 
Reed, MD (Saint Peters); New Jersey: Delaware Valley Pediatric Associates, PA (Lawrenceville), Chestnut Ridge Pediatric Associates (Woodcliff Lake); New Mexico: Albuquerque Pediatric Associates, Ltd (Albuquerque), Presbyterian Family Healthcare - Rio Bravo (Albuquerque), University of New Mexico Hospital (Albuquerque), First Step Pediatrics (Las Cruces); New York-1: Elmwood Pediatric Group (Rochester), Lewis Pediatrics (Rochester), Parkway Pediatrics (Rochester), Parkway Primary Care Clinic (Salamanca); New York-2: Sonia M Vinas, MD (Brooklyn); North Carolina: Carolinas Medical Center (Charlotte), Elizabeth Pediatrics (Charlotte), Novant Health, Eastover Pediatrics (Charlotte), Randolph Pediatrics (Charlotte), Matthews Children's Clinic, PA (Matthews); North Dakota: Altru Clinic (Grand Forks); Ohio: Bryan Medical Group (Bryan), Oxford Pediatrics \& Adolescents (Oxford), North Central Ohio Family Care Center, Inc (Galion); Pennsylvania: Laurel Health Center (Blossburg), Coudersport Pediatrics (Coudersport), Laurel Health Center (Mansfield), Pennridge Pediatric Associates (Sellersville), Laurel Health Center (Wellsboro); South Carolina: Palmetto Pediatrics \& Adolescent Clinic, PA (Columbia); Texas: Sarah L Helfand, MD (Dallas), Su Clinica Familiar (Harlingen), Family Medical Center (Midland), Rainbow Pediatric Clinic (Weslaco); Utah: Alpine Pediatrics (American Fork), John Weipert, MD (American Fork), Utah Valley Pediatrics, LC (American Fork), Michael Whiting, MD (American Fork), Utah Valley Pediatrics, LC - Cherry Tree Office (Orem), Pediatric Care, PC (Pleasant Grove), Utah Valley Pediatrics, LC - Physician Plaza (Provo), Utah Valley Pediatrics, LC - Provo North University Office (Provo), Uintah Basin Medical Center (Roosevelt), University South Main Public Health Center (Salt Lake City); Vermont: Green Mountain Pediatrics, PC (Bennington), University Pediatrics, UHC Campus (Burlington), Rebecca Collman, MD (Colchester), Essex Pediatrics (Essex Junction), Shelburne Pediatrics, Inc (Shelburne), Hagan \& Rinehart Pediatricians (South Burlington), Pediatric Medicine (South Burlington), Timber Lane Pediatrics (South Burlington), University Pediatrics (Williston); Virginia: Stonewall Pediatrics (Lexington); Washington: Northwest Pediatric Center (Centralia), Oakland Bay Pediatrics (Shelton), Yakima Neighborhood Health Services, Inc (Yakima); West Military: Naval Medical Center San Diego (San Diego); West Virginia: Marshall University Medical Center (Huntington), Grant Memorial Pediatrics (Petersburg); Wisconsin: Beloit Clinic SC (Beloit), Waukesha Medical Centers -
Muskego (Muskego), Waukesha Pediatric Associates (Waukesha); Wyoming: Jackson Pediatrics (Jackson).

\section{References}

1 Agency for Healthcare Research and Quality. Fact Sheet: Primary Care Practice-Based Research Networks. http:/ / www.ahrq.gov/research/pbrn/pbrnfact.htm [last accessed October 2007].

2 Pantell RH, Newman TB, Bernzweig J, Bergman DA, Takayama JI, Segal M, et al. Management and outcomes of care of fever in early infancy. JAMA 2004; 291:1203-1212.

3 Bernstein H, Spino C, Baker A, Slora E, Touloukian C, McCormick M. Postpartum discharge: do varying perceptions of readiness impact health outcomes? Ambulatory Pediatrics 2002; 2:388-395.

4 Taylor JA, Darden PM, Brooks DA, Hendricks JW, Wasserman RC, Bocian AB. The association between parental preferences and perceptions of barriers to vaccination and the immunization status of their children: a study from PROS and the National Medical Association. Pediatrics 2002; 110:1110-1116.

5 Mold JW, Roberts M, Aboshady HM. Prevalence and predictors of night sweats, day sweats, and hot flashes in older primary care patients: an OKPRN study. Annals of Family Medicine 2004; 2:391-397.

6 Vinson DC, Galliher JM, Reidinger C, Kappus JA. Comfortably engaging: which approach to alcohol screening should we use? Annals of Family Medicine 2004; 2:398-404.

7 Wasserman RC, Slora EJ, Bocian AB, Fleming GV, Baker AE, Pedlow SE, et al. Pediatric Research in Office Settings (PROS): a national practice-based research network to improve children's health care. Pediatrics 1998; 102:13501357.

8 Herman-Giddens ME, Slora EJ, Wasserman RC, Bourdony CJ, Bhapkar MV, Koch GG, et al. Secondary sexual characteristics and menses in young girls seen in office practice: a study from the Pediatric Research in Office Settings network. Pediatrics 1997; 99:505-512.

9 Wasserman RC, Croft CA, Brotherton SE. Preschool vision screening in pediatric practice: a study from the Pediatric Research in Office Settings (PROS) network. Pediatrics 1992; 89:834-838.

10 Darden PM, Taylor JA, Slora EJ, Hasemeier CM, Asmussen $\mathrm{L}$, Recknor JC, et al. Methodological issues in determining rates of childhood immunization in office practice: a study from Pediatric Research in Office Settings (PROS). Archives of Pediatrics and Adolescent Medicine 1996; 150:1027-1031.

11 Gardner W, Kelleher KJ, Wasserman R, Childs G, Nutting P, Lilienfeld $\mathrm{H}$, et al. Primary care treatment of pediatric psychosocial problems: a report from PROS and ASPN. Pediatrics 2000; 106:e44.

12 Beebe SA, Britton JR, Britton HL, Fan P, Jepson B. Neonatal mortality and length of newborn hospital stay. Pediatrics 1996; 98:321-335.

13 Brumfield CG, Nelson KG, Stotser D, Yarbaugh D, Patterson P, Sprayberry NK. 24-hour mother-infant discharge with a follow-up home health visit: results in a selected Medicaid population. Obstetrics and Gynecology 1996; 88:544-548. 
14 Cooper WO, Kotagal UR, Atherton HD, Lippert CA, Bragg $\mathrm{E}$, Donovan EF, et al. Use of health care services by inner city infants in an early discharge program. Pediatrics 1996; 98:686-691.

15 Edmonson MB, Stoddard JJ, Owens LM. Hospital readmission with feeding-related problems after early postpartum discharge of normal newborns. JAMA 1997; 278:299-303.

16 Liu LL, Clemens CJ, Shay DK, Davis RL, Novack AH. The safety of newborn discharge. The Washington State experience. JAMA 1997; 278:293-298.

17 Mandl K, Brennan T, Wise P, Tronick E, Homer C. Maternal and infant health effects of moderate reductions in postpartum length of stay. Archives of Pediatrics and Adolescent Medicine 1997; 151:915-921.

18 Soskolne EI, Schumacher R, Fyock C, Young ML, Schork A. The effect of early discharge and other factors on readmission rates of newborns. Archives of Pediatrics and Adolescent Medicine 1996; 150:373-379.

19 Kotagal UR, Atherton HD, Eshett R, Schoettker PJ, Perlstein $\mathrm{PH}$. Safety of early discharge for Medicaid newborns. JAMA 1999; 282:1150-1156.

20 Braveman P, Egerter S, Pearl M, Marchi K, Miller C. Early discharge of newborns and mothers: a critical review of the literature. Pediatrics 1995; 96:716-726.

21 Centers for Medicare and Medicaid Services. The Newborns' and Mothers' Health Protection Act. http://www.cms.hhs. gov / quarterlyproviderupdates/downloads/CMS2127F.pdf [last accessed February 2005].

22 Kimberly MB, Hoehn KS, Feudtner C, Nelson RM, Schreiner M. Variation in standards of research compensation and child assent practices: a comparison of 69 institutional review board-approved informed permission and assent forms for 3 multicenter pediatric clinical trials. Pediatrics 2006; 117:1706-1711.

23 Wolf LE, Zandecki J, Lo B. Institutional review board guidance on pediatric research: missed opportunities. Journal of Pediatrics 2005; 146:84-89.

24 Larson E, Bratts T, Zwanziger J, Stone P. A survey of IRB process in 68 US hospitals. Journal of Nursing Scholarship 2004; 36:260-264.

25 Butts AM, Alexander C. Use of health diaries with children. Nursing Research 1991; 40:59-61.

26 Berman ME. Health diaries in nursing research and practice. Image 1995; 27:147-152.

27 Richardson A. The health diary: an examination of its use as a data collection method. Journal of Advanced Nursing 1994; 19:782-791.

28 Holme CO. Incidence and prevalence of non-specific symptoms and behavioral change in infants under the age of two years. British Journal of Family Practice 1995; 45:65-69.

29 Roughmann KJ, Haggerty RJ. The diary as a research instrument in the study of health and illness behavior. Medical Care 1972; 10:143-163.

30 Barr RG, Kramer MS, Boisjoly C, McVey-White L, Pless IB. Parental diary of infant cry and fuss behavior. Archives of Disease in Childhood 1988; 63:380-387.
31 Schwartz J, Wypij D, Dockery D, Ware J, Zeger S, Spengler $\mathrm{J}$, et al. Daily diaries of respiratory symptoms and air pollution: methodologic issues and results. Environmental Health Perspectives 1991; 90:181-187.

32 Freer CB. Health diaries: a method of collecting health information. Journal of the Royal College of General Practitioners 1980; 30:279-282.

33 Yaffe R, Shapiro S, Fuchsberg RR, Rohde CA, Corpeno HC. Medical economics survey-methods study: cost effectiveness of alternative survey strategies. Medical Care 1978; 16:641659.

34 Verbrugge LM. Health diaries. Medical Care 1980; 28:73-95.

35 Promoting the Health of Newborns and Mothers through Postpartum Services: Final Report to Congress Mandated by the Newborns' and Mothers' Health Protection Act of 1996 (USA Public Law 104-240, Section 606).

36 Vollmer WM, Svetkey LP, Appel LJ, Obarzanek E, Reams P, Kennedy B, et al. Recruitment and retention of minority participants in the DASH controlled feeding trial. DASH Collaborative Research Group. Dietary Approaches to Stop Hypertension. Ethnicity and Disease 1998; 8:198-208.

37 Margitic S, Sevick MA, Miller M, Albright C, Banton J, Callahan $\mathrm{K}$, et al. Challenges faced in recruiting patients from primary care practices into a physical activity intervention trial. Preventive Medicine 1999; 29:277-286.

38 Bocian AB, Wasserman RC, Slora EJ, Kessel D, Miller R. The size and age-sex distribution of pediatric practice: a study from Pediatric Research in Office Settings (PROS). Archives of Pediatrics and Adolescent Medicine 1999; 153:9-14.

39 The American College of Obstetricians and Gynecologists. Research Page: Collaborative Ambulatory Research Network (CARN). http://www.acog.org/departments/dept_ notice.cfm?recno=16\&bulletin $=509$ [last accessed May 2006].

40 Swanson GM, Ward AJ. Recruiting minorities into clinical trials: toward a participant-friendly system. Journal of the National Cancer Institute 1995; 87:1747-1759.

41 Bell-Syer SEM, Klaber Moffett JA. Recruiting patients to randomized trials in primary care: principles and case study. Family Practice 2000; 17:187-191.

42 Walson PD. Patient recruitment: US perspective. Pediatrics 1999; 104:619-622.

43 Britton JR, Baker A, Spino C, Bernstein HH. Postpartum discharge preferences of pediatricians: results from a national survey. Pediatrics 2002; 110:53-60.

44 Barzi F, Woodward M. Imputations of missing values in practice: results from imputations of serum cholesterol in 28 cohort studies. American Journal of Epidemiology 2004; 160:3445 .

45 Escobar GJ, McCormick MC, Zupancic JAF, Coleman-Phox $\mathrm{K}$, Armstrong MA, Greene JD, et al. Unstudied infants: outcomes of moderately premature infants in the neonatal intensive care unit. Archives of Disease in Childhood. Fetal and Neonatal Edition 2006; 91:F238-F244.

46 Mitchell R, Shah M, Ahmad S, Rogers AS, Ellenberg JH. A unified web-based Query and Notification System (QNS) for subject management, adverse events, regulatory, and IRB components of clinical trials. Clinical Trials 2005; 2:61-71. 